\title{
Erratum to: Does a quality improvement campaign accelerate take-up of new evidence? A ten-state cluster-randomized controlled trial of the IHI's Project JOINTS
}

Eric C. Schneider ${ }^{1,2,3,7^{*}}$, Melony E. Sorbero ${ }^{1}$, Ann Haas $^{1}$, M. Susan Ridgely ${ }^{1}$, Dmitry Khodyakov ${ }^{1}$, Claude M. Setodji ${ }^{1}$, Gareth Parry ${ }^{4}$, Susan S. Huang ${ }^{5}$, Deborah S. Yokoe ${ }^{6}$ and Don Goldmann ${ }^{4}$

\section{Erratum}

Following the publication of the original article [1], it was brought to our attention that the title contained an error: "The Institute for Healthcare Improvement's Project JOINTS" was incorrectly included as "the Institute for Health Improvement's Project JOINTS".

The title has been corrected to read: "Does a quality improvement campaign accelerate take-up of new evidence? A ten-state cluster-randomized controlled trial of the IHI's Project JOINTS".

The corrected title has been included in this erratum and updated in the original article.

\footnotetext{
Author details

${ }^{1}$ RAND Corporation, Santa Monica, CA, USA. ${ }^{2}$ Division of General Medicine and Primary Care, Brigham and Women's Hospital, Boston, MA, USA. ${ }^{3}$ Department of Health Policy and Management, Harvard School of Public Health, Boston, MA, USA. ${ }^{4}$ Institute for Healthcare Improvement and Department of Pediatrics, Boston Children's Hospital, Boston, MA, USA. ${ }^{5}$ Division of Infectious Diseases and Health Policy Research Institute, University of California Irvine School of Medicine, Irvine, CA, USA. ${ }^{6}$ Division of Infectious Diseases, Brigham and Women's Hospital and Harvard Medical School, Boston, MA, USA. ${ }^{7}$ The Commonwealth Fund, One East 75th Street, New York, NY 10021, USA.
}

Received: 4 May 2017 Accepted: 4 May 2017

Published online: 10 May 2017

\section{Reference}

1. Schneider EC, et al. Does a quality improvement campaign accelerate take-up of new evidence? A ten-state cluster-randomized controlled trial of the $\mid \mathrm{HI}$ 's Project JOINTS. Implement Sci. 2017;12:51. doi:10.1186/s13012-017-0579-7.

\footnotetext{
*Correspondence: es@cmwf.org

${ }^{1}$ RAND Corporation, Santa Monica, CA, USA

2Division of General Medicine and Primary Care, Brigham and Women's

Hospital, Boston, MA, USA
} 